\title{
CONTRIBUIÇÕES DO COMPONENTE CURRICULAR ANATOMIA APLICADA A ENFERMAGEM
}

Anne Carolinne Marie dos Santos Gomes Anderson Felix dos Santos ${ }^{2}$

Jackson Soares Ferreira ${ }^{1}$

Denival Nascimento Vieira Júnior ${ }^{3}$

Josélio Soares de Oliveira Filho ${ }^{1}$

Waléria Bastos de Andrade Gomes Nogueira

\author{
http://orcid.org/0000-0001-8464-2585 \\ https://orcid.org/0000-0001-6622-3934 \\ https://orcid.org/0000-0001-8855-3512 \\ https://orcid.org/0000-0001-8813-0472 \\ https://orcid.org/0000-0002-4490-8075 \\ http://orcid.org/0000-0002-5208-108X
}

Objetivo: analisar sob a ótica discente as contribuições trazidas pela Anatomia Aplicada à Enfermagem enquanto componente curricular bem como a compreensão acerca das principais regiões anatômicas como embasamento para as práticas realizadas pelo enfermeiro a partir do conhecimento anatômico. Metodologia: estudo exploratório e descritivo com abordagem quanti-qualitativa, realizada com 122 acadêmicos de enfermagem, em uma instituição de ensino superior privada em João Pessoa - PB, utilizando o software IRAMUTEQ para o processamento dos dados qualitativos e Técnica de Análise de Conteúdo. Resultados: identificou-se que 98\% dos discentes entrevistados afirmaram que a Anatomia Aplicada à Enfermagem proporciona segurança durante procedimentos de enfermagem, bem como as principais estruturas anatômicas utilizadas durante a realização das técnicas baseadas em evidências. Conclusão: evidenciou-se a importância da Anatomia Aplicada à Enfermagem tal como o efetivo conhecimento apresentado pelos discentes sobre o domínio das principais regiões anatômicas, fundamentais para a realização de procedimentos de enfermagem.

Descritores: Anatomia; Enfermagem; Conhecimento; Terapêutica.

\section{CONTRIBUTIONS OF THE CURRICULUM COMPONENT ANATOMY APPLIED TO NURSING}

Objective: to analyze from the student's perspective the contributions brought by Applied Anatomy to Nursing as a curriculum component as well as the comprehension of the main anatomical regions as a basis for the practices performed by nurses based on anatomical knowledge. Methodology: exploratory and descriptive study with quantitative and qualitative approach, conducted with 122 nursing students, in a private higher education institution in João Pessoa, Paraiba, using the IRAMUTEQ software for qualitative data processing and Content Analysis Technique. Results: It was found that 98\% of the students interviewed stated that Anatomy Applied to Nursing provides safety during nursing procedures, as well as the main anatomical structures used during the execution of evidence-based techniques. Conclusion: the importance of Anatomy Applied to Nursing was noticed as well as the effective knowledge presented by the students about the mastery of the main anatomical regions, essential for the performing of nursing procedures.

Keywords: Anatomy Nursing; Knowledge; Therapeutics.

\section{CONTRIBUCIONES DE LAANATOMİA DEL COMPONENTE CURRICULAR APLICADAA LA ENFERMERİA}

Objetivo: analizar desde la perspectiva del alumno las contribuciones aportadas por la Anatomía Aplicada a la Enfermería como componente curricular, asi como la comprensión de las principales regiones anatómicas como base para las prácticas realizadas por las enfermeras basadas en el conocimiento anatómico. Metodologia: estudio exploratorio y descriptivo con enfoque cuantitativo y cualitativo, realizado con 122 estudiantes de enfermería, en una institución privada de educación superior en João Pessoa, Paraíba, utilizando el software IRAMUTEQ para el procesamiento cualitativo de datos y la técnica de análisis de contenido. Resultados: Se encontró que el $98 \%$ de los estudiantes entrevistados declararon que la Anatomía Aplicada a la Enfermería brinda seguridad durante los procedimientos de enfermería, asi como las principales estructuras anatómicas utilizadas durante la realización de técnicas basadas en evidencia. Conclusión: se evidenció la importancia de la anatomía aplicada a la enfermería, así como el conocimiento efectivo presentado por los estudiantes sobre el dominio de las principales regiones anatómicas, fundamentales para realizar procedimientos de enfermería.

Palabras clave: anatomia; enfermeira; conocimiento; terapéutica.

${ }^{1}$ Faculdade de Enfermagem Nova Esperança

${ }^{2}$ Universidade Federal da Paraiba

${ }^{3}$ Universidade Federal do Piaui

Autor Correspondente: Anne Carolinne Marie dos Santos Gomes - Email: anne_carolinne32@hotmail.com 


\section{INTRODUÇÃO}

A Anatomia Humana sempre se embasou na integração clínico-terapêutica, o que a tornou fundamental no currículo básico dos cursos de graduação em saúde ${ }^{(1-2)}$. É a partir desse conhecimento que é possibilitada uma futura compreensão sobre processos patológicos e problemas clíni$\cos ^{(3)}$.

Estudo identificou algumas competências importantes que são proporcionadas através da disciplina de anatomia humana como a compreensão estrutural, funcional e fisiológica dos sistemas e suas respectivas estruturas. Assim, faz-se necessária a associação dos conhecimentos morfológicos fornecidos pela disciplina aos fundamentos do aprendizado para a promoção de uma prática embasada cientificamente(3)

Enfatiza-se, a partir dessas ideações, o surgimento da Anatomia Aplicada, abordando os aspectos da estrutura e função do corpo humano indispensáveis à prática da enfermagem, associando as estruturas anatômicas aos procedimentos clínicos e exame físico durante consulta de enfermagem ${ }^{(4)}$.

Associa-se, nesta perspectiva, o componente curricular Anatomia Aplicada no curso de enfermagem às técnicas ministradas a partir do estudo de semiologia e semiotécnica em enfermagem. Deparando-se com técnicas a serem realizadas no âmbito da enfermagem em contato com o corpo humano, o estudante passará a relembrar dos conhecimentos básicos de Anatomia Humana, devendo então associá-la na prática clínica e nos procedimentos de enfermagem ${ }^{(5)}$

O entendimento do corpo a ser cuidado como elemento significativo da formação acadêmica e da profissão, nos revela originalmente a essência dos fundamentos que guiam a prática de enfermagem e fornece subsídios para discussão referente aos conhecimentos morfológicos, como elo interdisciplinar ao núcleo da área fundamental em enfermagem ${ }^{(5)}$.

Conhecer disciplinas fundamentais ao alicerce da biociência fornece sérias implicações nos padrões da prática do enfermeiro e, entre elas, garante a segurança do paciente, já que confere a esse profissional maior responsabilidade e competência em desenvolver estudos clínicos ${ }^{(3)}$.

Ante o exposto, entende-se que o estudante de enfermagem deve ser incentivado a construir conceitos e associações teóricas, práticas e clínicas referentes às disciplinas cursadas que the garantam a conexão entre os períodos e atendam aos desafios que emergiram na atenção à saúde. Acrescenta-se ainda que essa habilidade pode ser considerada como uma valiosa ferramenta na superação da fragmentação da prática educativa, a qual deveria fomentar os modelos fundamentados no processo de ação-reflexão e no olhar crítico do aprendiz(3).

Diante dessa discussão, questiona-se: quais as contribuições da disciplina Anatomia Aplicada à Enfermagem para a formação acadêmica, segundo a ótica discente? É, portanto, objetivo deste estudo, analisar sob a ótica discente as contribuições trazidas pela Anatomia Aplicada à Enfermagem enquanto componente curricular bem como a compreensão acerca das principais regiões anatômicas como embasamento para as práticas realizadas pelo enfermeiro a partir do conhecimento anatômico.

\section{METODOLOGIA}

\section{Tipo de estudo}

Realizou-se uma pesquisa do tipo exploratório-descritiva com abordagem quanti-qualitativa.

\section{Participantes do estudo}

A população do presente estudo foi composta por todos os alunos regularmente matriculados entre o quarto e oitavo semestre da graduação em enfermagem. Para definição da amostra, considerou-se erro amostral de 5\% e nível de confiança em 95\%, resultando em um quantitativo de 122 alunos. Para melhor estabelecer o perfil dos sujeitos participantes, adotou-se como critérios de inclusão: idade igual ou superior a 18 anos; já ter cursado a disciplina/ módulo referente a Anatomia Aplicada; e exclusão: alunos que não tenham interesse em participar ou estejam impossibilitados para tal. A escolha dos participantes abrangidos pelos critérios de inclusão se deu de forma aleatória.

\section{Local do estudo}

O estudo foi desenvolvido em uma Instituição de Ensino Superior Privada, situada na cidade de João Pessoa (PB). Esta escolha se deu em virtude de a Instituição adotar a disciplina "Anatomia Aplicada" como componente curricular obrigatório da graduação em enfermagem.

\section{Coleta de dados}

A coleta de dados ocorreu entre os meses de fevereiro e março de 2018. Para realização da coleta utilizou-se um questionário estruturado, elaborado segundo o objetivo, subdividido em duas partes, a primeira contendo perguntas referentes aos dados sociais, a segunda, sobre a temática pesquisada, com questionamentos relacionados a segurança na realização de procedimentos de enfermagem fornecida por intermédio do componente curricular Anatomia Aplicada à Enfermagem; bem como indagações 
inerentes aos principais órgãos e estruturas fundamentais para execução da profissão de enfermagem. Realizou-se a pesquisa conforme o funcionamento das aulas do curso de enfermagem, nos turnos manhã e noite, abordando-se de forma aleatória os acadêmicos e apresentando a finalidade do estudo, com posterior aceitação de participação por meio de assinatura do Termo de Consentimento Livre e Esclarecido (TCLE).

\section{Procedimentos de análise de dados}

Os dados quantitativos oriundos da coleta foram organizados em tabelas e discutidos à luz da literatura pertinente. Já os dados qualitativos, foram transcritos para word e transferidos para o bloco de notas para processamento pelo software IRAMUTEQ e após, foram analisados com a Técnica de Análise de Conteúdo, modalidade temática, proposta por Bardin(6).

\section{Procedimentos éticos}

Conforme o que pressupõe as diretrizes éticas que inerem a pesquisa envolvendo seres humanos, o estudo seguiu o que dispõe código de ética dos profissionais de enfermagem e a Resolução 466/12 do Conselho Nacional de Saúde, obtendo parecer favorável após apreciação do Comitê de Ética em Pesquisa sob protocolo № 04/2017, CAAE: 64393517.7.0000.5179.

\section{RESULTADOS}

Em sua maioria, os sujeitos participantes da pesquisa inserem-se no gênero feminino (63,9\%) e têm idade entre 18 e 24 anos de idade (40,9\%), cursando a partir do quinto período (81,7\%), como mostra a tabela 1.

Tabela 1 - Disposição das variáveis caracterizando os sujeitos do estudo. João Pessoa - PB, 2018.

\begin{tabular}{lll}
\hline VARIÁVEIS & $n$ & $\%$ \\
\hline Idade & 51 & \\
\hline $18-24$ & 49 & 40,9 \\
$25-34$ & 22 & 40,1 \\
35 ou mais & & 18,0 \\
\hline Periodo & 13 & \\
\hline 3 Periodo & 9 & 10,6 \\
4 ㅇ Período & 40 & 7,3 \\
5 o Periodo & 9 & 32,7 \\
6 Periodo & 33 & 7,3 \\
7 o Periodo & 18 & 27,0 \\
8 ㅇ Periodo & & 14,7 \\
\hline Gênero & 20 & 83,4 \\
\hline Masculino & 102 & 16,6 \\
Feminino & &
\end{tabular}

Quando indagados acerca da segurança que a disciplina Anatomia Aplicada à Enfermagem proporciona para a execução dos procedimentos em âmbito prático e profissional, a maioria dos discentes (98\%) afirmaram obter maior confiança a partir do ensinamento teórico-prático da disciplina.

Para melhor explicitar e justificar as respostas evocadas pelos discentes participantes, os discursos processados pelo software Iramuteq foram dispostos conforme Análise Fatorial de Correspondência - (Figura 1), gerando cinco categorias denominadas: Anatomia Instrumental (verde); Anatomia Aplicada como Subsídio à Prática Profissional (cor vermelha); Anatomia na Gênese do Cuidado (cinza), Prática Baseada em Evidências Anatômicas (cor roxa); e Conhecimento Teórico-Prático para a Supressão de erros (cor azul).

Figura 1 -Análise Fatorial de Correspondência (AFC), representando em classes os vocábulos evocados para responder ao questionamento entre o componente curricular e a prática profissional. João Pessoa, PB, Brasil, 2018.

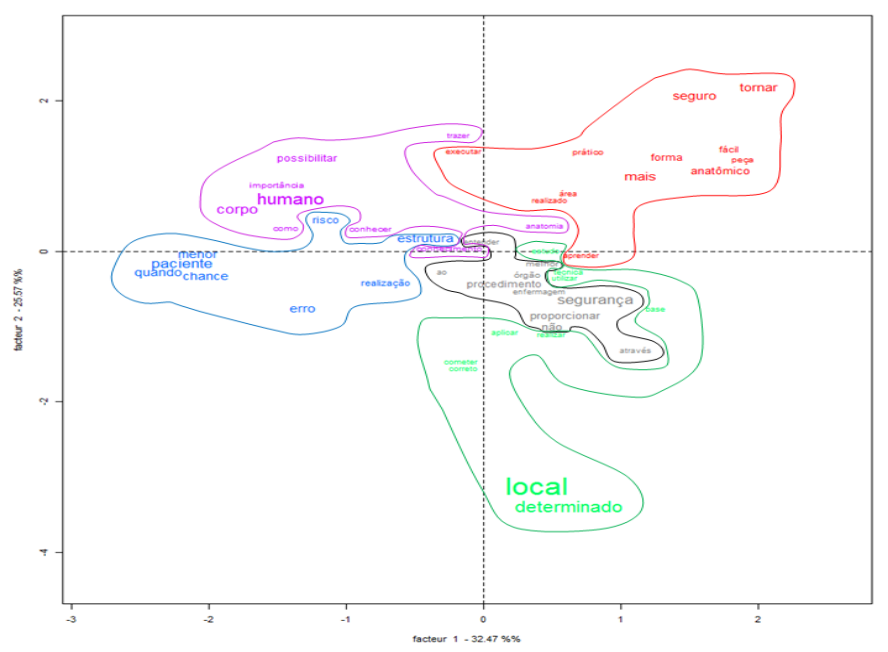

Fonte: Dados da pesquisa processados pelo IRAMUTEQ, 2018.

\section{Conhecimentos técnicos e científicos para a realização dos principais procedimentos de enfermagem}

Considerou-se o método "Nuvem de Palavras", para organizar e agrupar as palavras em diferentes tamanhos conforme a frequência que foram evocadas. Sendo assim, o mesmo torna-se o melhor método em detrimento dos demais disponibilizados pelo IRAMUTEO, no qual permite avaliar os conhecimentos sobre a relação entre a técnica de inserção da sonda vesical de demora (SVD) e as estruturas anatômicas subjacentes(7). 
Pode-se observar que o vocábulo "uretra" destacou-se como a estrutura mais citada pelos participantes, seguida de "bexiga", "óstio externo" e "interno" bem como as porções da "uretra", "membranosa", "esponjosa" e "prostática”.

Associando-se a estas palavras evocadas, a utilização das expressões "Genitália Masculina" e "Genitália Feminina", carregam consigo os vocábulos "pênis" como órgão "masculino" e a "vagina" como órgão "feminino".

Figura 2 - Sondagem Vesical. João Pessoa, PB, Brasil, 2018.

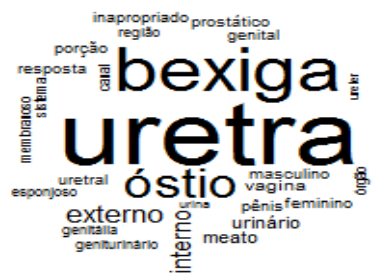

Fonte: Dados da pesquisa processados pelo IRAMUTEQ, 2018.

Para analisar as estruturas anatômicas e regiões assumidas como referência para a inserção de uma sonda nasogástrica e nasoentérica (SNG/SNE), utilizou-se a análise de similitude, como método para evidenciar os vocábulos mais citados pelos participantes. Esta expressa o essencial da estrutura de associações existentes do conjunto dos elementos de representação, de modo que permite ligações entre as diferentes palavras citadas pelos entrevistados resultando em possibilidades de comparação(7) $^{(7)}$

É possivel considerar que a evocação de maior relevância conforme o discurso é o "estômago", ocupando o centro da rede de palavras na Figura 3, cujo qual relaciona-se com o "nariz". O termo "lóbulo" que está segmentado a partir de "orelha", "processo" ou "ponta", correlacionando-se com "xifoide". A partir da palavra "esôfago", pode-se visualizar a "traqueia".

Interligados ao órgão estômago, pode-se visualizar os vocábulos como: "óstio cárdico", "duodeno", "jejuno", "intestino delgado"; bem como intestino "grosso". O "íleo", apesar de relacionar-se ao intestino delgado e ainda ser citado por alguns alunos questionados, não é alcançado na passagem de sonda nasoentérica.

Figura 3 - Sondagem Nasogástrica/Nasoentérica. João Pessoa, PB, Brasil, 2018

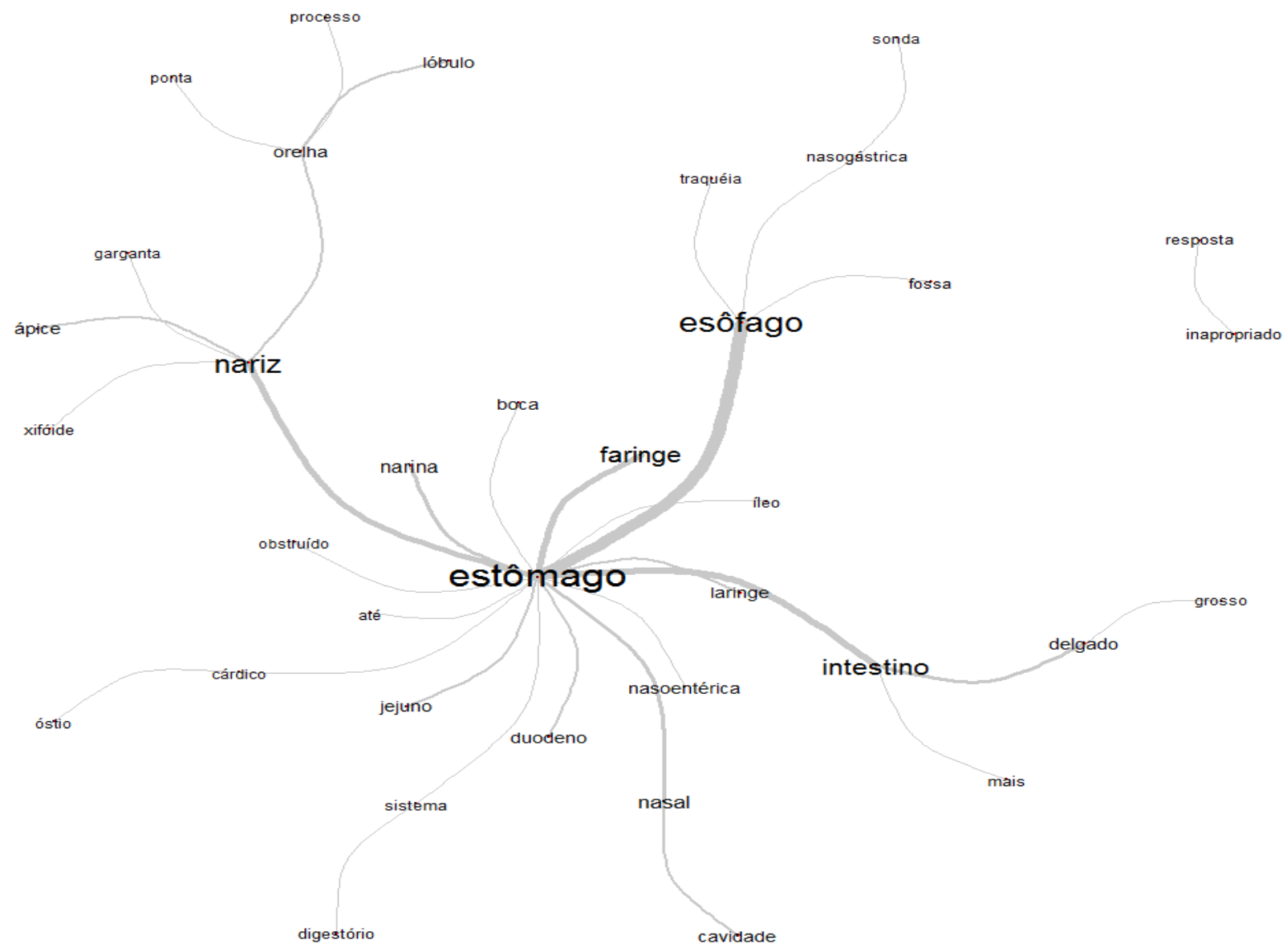

Enferm. Foco 2019; 10 (6): 93-98 | 95 
Fonte: Dados da pesquisa processados pelo IRAMUTEQ, 2018

\section{DISCUSSÃO}

A Anatomia Aplicada à Enfermagem durante o curso, é o componente curricular referência para a aprendizagem da realização dos principais procedimentos de enfermagem, sendo necessário o conhecimento das principais estruturas do corpo humano para aplicabilidade das técnicas de enfermagem. Caracteriza-se a maior parte dos discentes como estudantes do quinto período em diante, sendo tal característica semelhante a estudo ${ }^{\left({ }^{(8)}\right.}$ que evidenciou discentes de períodos avançados como reconhecedores da necessidade da anatomia para subsidiar a técnica em busca de achados clínicos.

A maioria dos discentes desta pesquisa afirma a relevância do componente curricular Anatomia Aplicada à Enfermagem para a promoção da segurança durante os procedimentos de enfermagem. O conhecimento dos componentes curriculares iniciais dos cursos de graduação em enfermagem é indispensável para garantir a segurança do paciente, propiciando ao profissional maior aptidão para desenvolvimento de estudos clínicos ${ }^{(9)}$.

A partir do conhecimento anatômico, o enfermeiro é capaz de exercer funções complexas, ampliando seu conhecimento científico, promovendo intervenções especializadas para cada paciente ${ }^{(10)}$. Deve-se manter a aquisição dos conhecimentos durante o cursar da anatomia como subsídio para demais componentes curriculares como semiotécnica e semiologia e no âmbito profissional na realização do raciocínio clínico e distinção da normalidade(11-12)

A partir dos dados deste estudo foi possivel inferir que, conhecer a anatomia proporciona maior autoconfiança ao estudante de enfermagem que, enquanto enfermeiro após sua formação executará os procedimentos de enfermagem baseados nos conhecimentos e motricidade fina, acarretando consequentemente em uma melhor assistência, sendo o enfermeiro capaz de transmitir segurança e proteção ao paciente, tornando-o confiante de que o mesmo proporcionará melhorias à sua saúde.
Pôde-se identificar as estruturas anatômicas como base para a realização da sondagem vesical, nasogástrica e nasoentérica. Identifica-se a partir deste estudo a imprescindível aplicação de um componente curricular voltado para as estruturas anatômicas associando-as à prática clínica, nas graduações de enfermagem.

O cateterismo vesical, que consiste em uma atividade privativa do enfermeiro ${ }^{(13)}$, caracteriza-se um procedimento estéril para fins terapêuticos, no qual facilita a entrada de microorganismos no trato urinário. Para tanto, exige-se capacitação, conhecimento e habilidades durante a realização e manipulação, consubstanciando na redução das complicações e infecções do sistema urinário(14-15)

A partir de estudo, pôde-se identificar o conhecimento deficiente quanto à passagem e manipulação do cateter (16). Além disso, outra pesquisa identificou que os protocolos das técnicas de cateterismo adequadas para prevenção de infecções do trato urinário de determinadas instituições de saúde encontraram-se inadequa$\operatorname{dos}^{(17)}$

Concebe-se, com isso, a preocupação do conhecimento anatômico para a inserção adequada da sonda vesical, adotando a prática baseada em evidências ${ }^{(18)}$.

A uretra é um tubo muscular que conduz a urina do "óstio interno" da uretra a partir da bexiga até o óstio externo da uretra para o meio externo. Encontra-se, o óstio interno da uretra em contato direto com a bexiga, permitindo a passagem da urina para a uretra, chegando ao óstio externo da uretra localizado na glande do pênis, ao que possui "órgão genital" considerado "masculino" e no vestíbulo da vagina, ao que possui órgão genital conceituado como "feminina", possuindo cerca de 4 centí$\operatorname{metros}^{(4)}$.

Notou-se no estudo que parte da amostra possui conhecimento apenas sobre três, das quatro porções encontradas na uretra masculina, que possui de 18 a $22 \mathrm{~cm}$ de comprimento podendo ter maiores ou menores variações de acordo com o tamanho do pênis sendo estas a "porção prostática, porção membranácea e porção esponjosa"(4). Destaca-se que, a região não citada em nenhum momento foi a parte intramural da uretra. 
Salienta-se tal oposição de ideias, que pode ser explicada segundo as literaturas de referência utilizadas para o desenvolvimento do componente curricular nesta instituição, cuja as mesmas detêm a subdivisão da uretra em apenas três partes.

Entende-se a partir de conceito da Sociedade Brasileira de Anatomia - SBA(19), que a uretra masculina é subdivida em quatro partes: a intramural que compreende a região que está no músculo detrusor da bexiga; a prostática na região da próstata; parte membranácea que atravessa a membrana do períneo; e, esponjosa que se estende pelo corpo esponjoso do pênis. Ratifica-se por Dangelo e Fattini(20) que na publicação de seu livro "Anatomia humana sistêmica e segmentada", apresentou a uretra masculina subdividida em quatro partes, acima citadas.

Percebe-se que, quando existe a correlação entre partes anatômicas e o conhecimento técnico científico de determinado procedimento, como a sondagem, torna-se possivel garantir a segurança na prestação da assistência, diminuindo infecções do trato urinário e complicações para o paciente(21).

Após a devolutiva do estudo para a instituição e professores do componente curricular Anatomia Aplicada à Enfermagem e Anatomia Humana, ficarão à critério acerca da utilização da nomenclatura baseada na Sociedade Brasileira de Anatomia(19) e Dangelo e Fant$\operatorname{tini}{ }^{(20)}$

Dar-se a introdução da SNG/SNE a partir do "nariz" passando por uma das "narinas" seguido da "cavidade nasal", "faringe" - que podemos considerar como um pequeno tubo e que faz parte do sistema respiratório bem como digestório - e adiante encontramos o "esôfago", o "óstio cárdico" delimitando o final do esôfago e o início do estômago, quanto que no final do estômago encontra-se outra estrutura denominada de esfíncter do piloro, que delimita o início da primeira porção do "intestino delgado", o “duodeno". Quando o paciente apresenta risco de refluxo esofágico que levaria à possível broncoaspiração, prefere-se a alimentação jejunal ${ }^{(22)}$, ou seja, a segunda porção do intestino delgado, o "jejuno".

Assim, pode-se observar diante dos resultados que, ao relacionar a anatomia humana com aplicabilidade da semiologia e semiotécnica em enfermagem, torna-se possível a associação dos conteúdos teóricos e práticos pelo profissional de enfermagem, con tribuindo para assistência de enfermagem adequada e segura.

\section{Limitação do estudo}

Há necessidade do conhecimento da anatomia humana por parte dos cursos de graduação das ciências da saúde, no entanto, a limitação deste estudo refere-se à escassez de manuscritos que enfatizem esta importância.

\section{Contribuição do estudo para a prática}

Este estudo coopera para possiveis melhorias nas bases de ensino anatômicas voltadas para o profissional de enfermagem. Denota-se ainda a necessidade de estudos voltados a avaliação do componente curricular Anatomia Aplicada à Enfermagem, da Anatomia Humana dentro das universidades, bem como a qualidade do ensino.

\section{CONCLUSÃO}

A partir das investigações realizadas nos discursos dos graduandos de enfermagem, observou-se que a disciplina de Anatomia Aplicada à Enfermagem é de fundamental importância para a formação de um profissional qualificado. Afirma-se que existe a necessidade do maior enfoque nas práticas clínicas, sendo este o objetivo geral da disciplina enquanto parte do conteúdo programático de algumas graduações de enfermagem. Com isso, enfatiza-se a importância da interação do estudo da semiologia e semiotécnica com as bases anatômicas para proporcionar uma assistência sistematizada ao futuro enfermeiro.

Avaliando, portanto, o aprendizado e a satisfação da disciplina por meio deste, os resultados foram satisfatórios, constando como maioria os participantes que apresentou domínio das principais regiões anatômicas, fundamentais para realização de procedimentos de enfermagem e que o insuficiente conhecimento da disciplina pode implicar em consequências ruins para a saúde do indivíduo. 


\section{REFERÊNCIAS}

1. Papa V, Vaccarezza M. Teaching anatomy in the XXI century: new aspects and pitfalls. Sci World J. [Internet] 2013 [cited 2019 Apr 20]

1(5). Available from: https://www.ncbi.nlm.nih.gov/pmc/articles/ PMC3842041/

2. Alsaid B. Slide shows vs graphic tablet live drawing for anatomy teaching. Morphologie [Internet] 2016 [cited 2019 Apr 20]; 100 (331). Available from: https://www.sciencedirect.com/science/article/pii/ S1286011516300248?via\%3Dihub

3. Oliveira RCE, Tinôco JDS, Delgado MF, Andriola IC, Silva CMB, Lira ALBC. Estratégia educativa no ensino de anatomia humana aplicada à enfermagem. Av enferm. [Internet] 2018 [cited 2019 Apr 20]; 36(1): 31-39. Available from: http://www.scielo.org.co/pdf/aven/v36nl/0121-4500aven-36-01-00031.pdf

4. Moore KL, Dalley AF, Agur AMR. Anatomia orientada para a clínica. 7th ed. Rio de Janeiro: Guanabara Koogan; 2014

5. Silva PS, Dias SL, Henriques JCF, Soares EF, Figueiredo NMA. Discurso sobre anatomia humana no currículo integrado de enfermagem. Rev. pesqui. cuid. fundam. [Internet] 2013 [cited 2019 abr 4]; 5(1). Available from: http://www.seer.unirio.br/index.php/cuidadofundamental/article/ view/1845/pdf_695

6. Bardin L. Análise de Conteúdo. São Paulo: Edições 70; 2011.

7. Camargo BV, Justo AM. IRAMUTEQ: um software gratuito para análise de dados textuais. Temas psicol. [Internet] 2013 [cited 2019 Apr 26]; 21(2): 513-518. Available from: http://pepsic.bvsalud.org/scielo. php?script=sci_arttextEpid=S1413-389X2013000200016

8. Santos AF, Ferreira MGN, Pordeus ACL, Moreira LSM, Souto CGV Maia CMAFG. Avaliação prática em anatomia numa perspectiva discente. Rev Nova Esperança. [Internet] 2018 [cited 2019 Apr 30]; 16(3): 33-42. Available from: http://www.facene.com.br/wp-content/ uploads/2018/12/Vol-16-n3-2018.pdf

9. Mannix J, Wilkes L, Jackson D. Marking out the clinical expert/clinical leader/clinical scholar: perspectives from nurses in the clinical arena. BMC Nurs. [Internet]. 2013 [cited 2019 May 15]; 12(12). Available from: https://bmcnurs.biomedcentral.com/articles/10.1186/1472-6955-12-12

10. Salbego C, Oliveira EMD, Silva MAR, Bugança PR. Percepções acadêmicas sobre o ensino e a aprendizagem em anatomia humana. Rev bras educ méd. [Internet] 2015 [cited 2019 May 20]; 39(1): 23-31. Available from: http://www.scielo.br/pdf/rbem/v39nl/1981-5271rbem-39-1-0023.pdf

11. Cocce ALR, Silveira LM, Góes FSN, Souza ALT, Stabile AM. O ensino da anatomia nas escolas de enfermagem: um estudo descritivo. Arq ciênc saúde. [Internet] 2017 [cited 2019 May 26]; 24(4): 08-13. Available from: http://www.cienciasdasaude.famerp.br/index.php/racs/article/ view/818/721

12. Lira ALBC, Fernandes MICD, Costa IA, Silva RSC. Estratégia de
Aprimoramento do Ensino do Exame Fisico em Enfermagem. Enferm Foco. [Internet] 2015 [cited 2019 Aug 22]; 6 (1/4): 57-61. Available from: http://revista.cofen.gov.br/index.php/enfermagem/article/ view/578/260

13. Conselho Federal de Enfermagem. Resolução COFEN no 0450 de 11 de dezembro de 2013. Normatiza o procedimento de Sondagem Vesical no âmbito do Sistema Cofen / Conselhos Regionais de Enfermagem. 2013 [cited 2019 Aug 22]. Available from: http://www.cofen.gov.br/resolucaocofen-no-04502013-4_23266.html

14. Amaral DM, Coropes VBAS, Paula CL, Vidal MLB. Pós-operatório de vulvectomia e cateterismo vesical de demora: revisão integrativa. Rev enferm UFPE. [Internet] 2017 [cited 2019 May 25]; 11(10): 3948-57. Available from: https://periodicos.ufpe.br/revistas/revistaenfermagem/ article/view/14283/24358

15. Gomes AC, Carvalho PO de, Lima ETA, Gomes ET, Valença MP. Cavalcanti ATA. Caracterização das infecções relacionadas à assistência à saúde em unidade de terapia intensiva. Rev enferm UFPE. [Internet] 2014 [cited 2019 May 05]; 8(6): 1577-85. Available from: https://periodicos. ufpe.br/revistas/revistaenfermagem/article/viewFile/9848/10059

16. Bardsley A. ISC in women following urogynaecologic surgery. $\mathrm{Br} \mathrm{J}$ Nurs. 2015; 24(18). Doi: https://doi.org/10.12968/bjon.2015.24.Supl8.S6

17. Chaves NMO, Moraes CLK. Controle de infecção em cateterismo vesical de demora em unidade de terapia intensiva. Rev enferm Cent.Oeste Min. [Internet] 2015 [cited 2019 May 5]; 5(2): 1650-1657. Available from: https://docs.google.com/viewerng/viewer?url=http://www.seer. ufsj.edu.br/index.php/recom/article/viewFile/773/867

18. Andrade VLF, Fernandes FAV. Acesso à assistência cirúrgica: desafios e perspectivas. Rev Latino-Am Enfermagem. [Internet] 2016 [cited 2019 Jun 6]; 24. Available from: http://www.revistas.usp.br/rlae/article/ view/113358/111322

19. Sociedade Brasileira de Anatomia (SBA). Terminologia Anatômica. 1th ed. São Paulo: Manole: 2001

20. Dangelo JG, Fattini CA. Anatomia humana sistêmica e segmentar.3th ed. Revista. São Paulo: Editora Atheneu; 2011.

21. Merces MC, Carvalho MAM, Araújo PRS, Queiroz AB, Silva BSM, Sousa MNM, Servo MLS. A prática do(a) enfermeiro(a) na inserção do cateter de Folley em pacientes de unidade de terapia intensiva: limites e possibilidades. Rev epidemiol controle infecç. [Internet]. 2013 [cited 2019 Jun 6]; 3(2): 55-61. Available from: http://www.fufs.edu.br/admin/ anexos/23-09-2013_14_49_45_.pdf

22. Potter P. Perry AG. Fundamentos de Enfermagem. 8th ed. Rio de Janeiro: Elsevier; 2013

RECEBIDO: 05/08/2019

ACEITO: 09/10/2019 